\title{
Dinâmica do potencial matricial em substratos de pinus e coco sob ação da capilaridade
}

\section{Carlos Vinícius G Barreto'; Roberto Testezlaf ${ }^{2}$; Conan A Salvador ${ }^{3}$}

${ }^{1}$ INPI, R. São Bento 1, $8^{\circ}$ andar, centro, 24246-170 Rio de Janeiro; ${ }^{2}$ UNICAMP-FEAGRI, Cidade Universitária Zeferino Vaz s/n ${ }^{\circ}$, 13085-970 Campinas-SP; cvbarreto@bol.com.br; bob@feagri.unicamp.br; ${ }^{3}$ USP-ESALQ, Depto. Eng. Biossistemas; conanayade@ yahoo.com.br

\section{RESUMO}

O tamanho médio de partículas e a porosidade dos substratos condicionam as propriedades matriciais, interferindo na capacidade de retenção e de transmissão da água no meio. O conhecimento desses atributos é fundamental em processos de irrigação por capilaridade, para que o molhamento atinja as camadas superiores dos recipientes com níveis de tensão de água facilmente disponível. O presente trabalho teve como objetivo estudar a ascensão de água por capilaridade para determinar a posição mais apropriada do nível de saturação na ascensão capilar em recipientes com substratos de coco e pinus, de textura grossa e fina. Foram efetuados experimentos avaliando a ascensão de água por capilaridade em colunas segmentadas preenchidas com os substratos. Os valores de umidade em cada segmento foram calculados gravimetricamente e relacionados aos de tensão estimados pela curva de tensão dos substratos. Os substratos com textura fina apresentaram melhor elevação de água por capilaridade, com melhor elevação da umidade em níveis de tensão de água disponível. O substrato de coco fino apresentou água disponível em todo o perfil do recipiente, enquanto o de pinus apresentou as camadas superiores do recipiente com água retida em tensões abaixo do ponto de murcha permanente. O substrato fino de coco apresentou os melhores resultados para aplicação na irrigação por capilaridade, permitindo recomendar o seu uso com o nível de saturação posicionado a cinco centímetros do fundo do recipiente por quinze minutos.

Palavras-chave: Água disponível, subirrigação, textura de substratos.

\begin{abstract}
Dynamics of matric potential on substrates of pine and coconut under the action of capillarity

The average particle size and porosity of substrates affects the matrix properties of root growth media, interfering in water holding and water conductivity capacity through substrates. The knowledge of these attributes is essential in capillary irrigation processes to allow the wetting front reaches upper layers under easily available water tension. The present work had the objective to study the water rise by capillary processes to determine the most favorable position of the saturation level for contained plants for two substrates: coconut fiber and pine bark, with two grain particle sizes: fine and coarse textures. Water rise trails were carried out in segmented columns filled with substrates. The water content in each column segment was estimated in weight basis and related to the values of water tension estimated by the water tension curve for each evaluated substrate. The results showed that fine substrates presented higher water rise with adequate levels of easily available water. The fine coconut substrate presented easily available water in the entire substrate column, unlike pine bark substrate that showed the upper layer with moisture below the wilting point. The fine coconut substrate showed the best results for capillary application, allowing to recommend irrigation schedule using the saturation level at $5 \mathrm{~cm}$ during 15 minutes.
\end{abstract}

Keywords: Easily available water, subirrigation, root growth media.

\section{(Recebido para publicação em 23 de novembro de 2010; aceito em 24 de janeiro de 2012)} (Received on November 23, 2010; accepted on January 24, 2012)

$\mathrm{O}$ s substratos de turfa, pinus, tortas de filtro ou de outros materiais orgânicos utilizados na produção agrícola em recipientes apresentam diferentes características físicas como porosidade, aeração ou capacidade de retenção de água (Verdonk et al., 1983). A natureza desses substratos determina diferenças nos processos de molhamento e secagem, sendo observada histerese menor naqueles de pinus (Naasz et al., 2005). $\mathrm{O}$ conhecimento do valor do potencial mátrico do substrato permite a inferência da sua umidade atual e a estimativa da condutividade hidráulica (Caron \& Elrick, 2005). O monitoramento do potencial mátrico possui a vantagem de ser mais preciso do que as técnicas de pesagens em manejo hídrico, pois os substratos apresentam variações de densidade, porosidade e distribuição de partículas (Landis, 1989). Adicionalmente, o conhecimento do potencial mátrico do substrato torna possível a determinação da tensão ótima para irrigação, evitando o estresse hídrico na planta (Raviv et al., 1993). O desempenho da ascensão de água nos substratos é inversamente proporcional ao estresse hídrico vegetal (Caron et al., 2005a). Assim sendo, a informação dessa propriedade hídrica é essencial para a otimização da irrigação por capilaridade. Neste sentido, substratos com predominância de partículas finas e, conseqüentemente com teor de água disponível e ascen- são capilar adequados favoreceriam a hidratação vegetal. Segundo Bunt (1983), substratos com texturas com partículas grosseiras acima de $25 \%$ da sua constituição apresentam reduzida fração de água facilmente disponível. Segundo De Kreij \& Straver (1988), quando é empregado manejo hídrico com freqüente aplicação de água por capilaridade, é desejável que os substratos apresentem teores mais elevados de aeração o que poderia contribuir para o aumento da produção. De acordo com Patel et al. (2000), a concentração de sais em meio de crescimento de raízes, como substratos, é dependente do posicionamento da frente de saturação e do tempo de sua atuação no sistema 
solo-planta. Estes autores afirmam que a presença mais elevada da posição da frente de saturação (ou menores níveis de tensão) determinaria a diluição dos sais nesse meio, reduzindo as suas concentrações. Portanto, o conhecimento dos níveis de energia na qual a água é retida na frente de molhamento devido à presença de uma superfície de água, é um fator importante e essencial para condução do manejo hídrico nas plantas cultivadas sob irrigação por capilaridade. Dessa forma, o presente trabalho teve por objetivo estudar a variação do potencial matricial dos substratos de coco e de pinus durante o processo de elevação de água por capilaridade e definir a altura de ascensão da água.

\section{MATERIAL E MÉTODOS}

Os experimentos foram realizados nos Laboratórios de Solos e no de Hidráulica e Irrigação da Faculdade de Engenharia Agrícola da Unicamp, de dezembro de 2005 a outubro de 2006. Foram utilizados os substratos de coco (C) e de pinus (P), com predominância de partículas finas (F) e grosseiras (C), denominados respectivamente como tratamentos CF, CC, PF e PC. A composição granulométrica de cada substrato é demonstrada na Tabela 1. A umidade inicial dos substratos era: $\mathrm{PF}=0,29$ $\mathrm{cm}^{3} / \mathrm{cm}^{3} ; \mathrm{PC}=0,28 \mathrm{~cm}^{3} / \mathrm{cm}^{3} ; \mathrm{CF}=0,27$ $\mathrm{cm}^{3} / \mathrm{cm}^{3}$ e a do $\mathrm{CC}=0,07 \mathrm{~cm}^{3} / \mathrm{cm}^{3}$. As densidades globais e de partículas dos substratos em $\mathrm{kg} / \mathrm{m}^{3}$, foram respectivamente; $\mathrm{PF}=266,8$ e 938,$9 ; \mathrm{PG}=268,8$ e 644,$1 ; \mathrm{CF}=199,9$ e 684,9 e $\mathrm{CG}=77,4$ e 600,6 .

Os substratos foram acondicionados em colunas experimentais de $60,25 \mathrm{~cm}^{3}$ construídas em PVC DN25 com $10 \mathrm{~cm}$ de altura útil, segmentados em anéis de dois centímetros $(\mathrm{cm})$, com a umidade recomendada pelo fabricante. A técnica adotada foi desenvolvida por Goebel et al. (2004), adaptada para determinação da ascensão capilar de água em solos. A principal adaptação foi a utilização de cilindros construídos a partir de tubos de PVC DN 25, com 10,3 cm de altura, segmentados em cinco anéis unidos com fita adesiva. Exceto o anel da base, todos os outros tinham dois centímetros altura; o anel da base pos- suía três milímetros ( $\mathrm{mm})$ a mais que os demais, para permitir o contato com a água na formação de zona de saturação (Figura 1). Na parte inferior deste anel foi fixado um tecido utilizando-se um elástico para evitar a perda de substrato. A capacidade útil de toda a coluna era de $60,25 \mathrm{~cm}^{3}$, com $12,05 \mathrm{~cm}^{3}$ para cada anel, inclusive para o primeiro, pois o volume adicional de $1,8 \mathrm{~cm}^{3}$ de volume decorrentes da sua maior altura, não foi amostrado (Figura 1).

No preenchimento dos cilindros com os substratos foi utilizado o método proposto por Yeager (1995) e Pire \& Pereira (2003). Para o preenchimento das colunas com o substrato úmido, foram necessários quatro impactos para o recipiente ficar totalmente cheio.

Os cilindros com os substratos foram colocados em uma bandeja de 20 x 30 cm que permitia a saturação das amostras pela manutenção de uma lâmina constante com $3 \mathrm{~mm}$ de água, formando uma zona de saturação interna no cilindro na região de contato com a água. A ascensão de água por capilaridade ao longo do tempo no cilindro foi determinada para os seguintes tempos de saturação: $0,25,0,5,1,2,4,8,16$ e 24 horas. Foram utilizadas 10 repetições por substrato, com os tratamentos dispostos no delineamento inteiramente casualizado. As determinações de umidade de cada amostra segmentada de substrato foram realizadas gravimetricamente, com base na massa do substrato seco, para cada um dos tempos de saturação avaliados.

Para obtenção dos valores da umidade volumétrica do substrato, os dados obtidos nas determinações segmentadas do substrato, foram multiplicados pelo valor da densidade média dos anéis. Com os dados de umidade volumétrica de cada anel foram estimados os valores do potencial matricial utilizando da equação ajustada de van Genuchten (1980).

Para a determinação da curva de retenção de água característica dos substratos, foram empregados anéis de aço inoxidável dispostos em mesa de tensão, empregando-se 3 repetições por substrato. Os substratos foram acondicionados em anéis de $10 \mathrm{~cm}$ (altura) por $20 \mathrm{~cm}$ (diâmetro), com o fundo fechado por um tecido poroso. As densidades utilizadas para o preenchimento das colunas e dos anéis de aço inoxidável foram de $0,18 \mathrm{~g} / \mathrm{cm}^{3}$ para os substratos de pinus fino e grosseiro, e de $0,07 \mathrm{~g} / \mathrm{cm}^{3}$ para o substrato fino de coco, e $0,06 \mathrm{~g} / \mathrm{cm}^{3}$ para o substrato grosseiro de coco. Estes valores de densidades são próximos aos utilizados em substratos empregados para a produção comercial e foram obtidos durante o preenchimento dos cilindros e dos anéis pela compactação com 4 golpes. A curva de retenção foi determinada empregando-se as tensões de $10,20,30,40,50$ e $60 \mathrm{~cm}$ de coluna de água (cm.c.a). Os valores de 70 até $100 \mathrm{~cm} . c . a$. foram extrapolados pela equação de van Genuchten (1980). Os valores da curva de retenção foram ajustados de acordo com van Genuchten (1980), conforme se observa na equação a seguir:

$$
\theta(\%)=\theta_{R}+\frac{\left(\theta_{S}-\theta_{R}\right)}{\left[1+\left(\alpha \times \Psi_{m}\right)^{n}\right]^{m}} X 100
$$

Onde $\mathrm{m}, \mathrm{n}$ e $\alpha=$ parâmetros de ajuste da curva; Or: Umidade residual $\left(\mathrm{cm}^{3} /\right.$ $\left.\mathrm{cm}^{3}\right)$; Os: Umidade saturada $\left(\mathrm{cm}^{3} / \mathrm{cm}^{3}\right)$.

Com os valores de umidade na coluna (coluna da Figura 1C), em que cada anel possui posição conhecida, correspondeu-se os valores de umidade de cada anel com seus respectivos valores de tensão, e assim, interpolaram-se os valores de $0,10,50$ e 100 cm.c.a na coluna de anéis segmentados. Estas posições representam os limites para saturação de água, espaço de aeração, água facilmente disponível e água tamponante (De Boodt et al.,1974). Os oito pontos obtidos para cada tensão de água na relação altura $\mathrm{x}$ tempo foram ajustados por meio de regressão não-linear calculada pelo programa Origin 8.1 , indicando a posição de cada nível de energia ao longo do tempo na coluna.

A água disponível para a coluna de substrato foi calculada com base no cômputo do volume de água excedente, retido a tensões abaixo de 100 cm.c.a. para cada anel da coluna de substrato. A soma do volume de água de cada anel foi dividida pelo volume da coluna, 60,25 $\mathrm{cm}^{3}$, obtendo, assim, o valor em $\Theta$.

\section{RESULTADOS E DISCUSSÃO}

\section{Curvas características de retenção}


Tabela 1. Distribuição percentual do tamanho de partículas para os substratos fino de pinus (PF), grosseiro de pinus (PG), fino de coco com componentes granulares (CF-G) e fibrosos (CF-F) e grosseiro de coco de componentes granulares (CG-G) e fibrosos (CG-F) (particle size distribution in percentage, for fine pine bark substrate $(\mathrm{PF})$, coarse pine bark substrate (CG), fine coconut substrate with granular components (CF-G) and fibrous components (CF-F) and coarse coconut with granular particles (CG-G) and fibrous (CG-F)). Campinas, FEAGRI, 2006.

\begin{tabular}{lccccccc}
\hline \multirow{2}{*}{ Substrato } & \multicolumn{7}{c}{ Massa retida na peneira (g) } \\
\cline { 2 - 8 } & $\mathbf{< 0 , 2 5}$ & $\mathbf{0 , 2 5}$ & $\mathbf{0 , 5}$ & $\mathbf{1}$ & $\mathbf{2}$ & $\mathbf{4 , 7}$ & $\mathbf{6 , 3}$ \\
\hline PF & 14,79 & 14,40 & 30,20 & 23,70 & 16,68 & 0,14 & 0,09 \\
PG & 11,68 & 10,86 & 22,44 & 19,54 & 31,76 & 2,58 & 1,15 \\
CF-G & 11,68 & 19,10 & 37,61 & 24,30 & 5,43 & 0,31 & 0,72 \\
CF-F & 0 & 0,03 & 0,31 & 0,77 & 0 & 0 & 0 \\
CG-G & 6,73 & 9,32 & 15,82 & 11,63 & 0 & 0 & 0 \\
CG-F & 2,69 & 3,11 & 8,24 & 4,41 & 5,61 & 0 & 32,46 \\
\hline
\end{tabular}

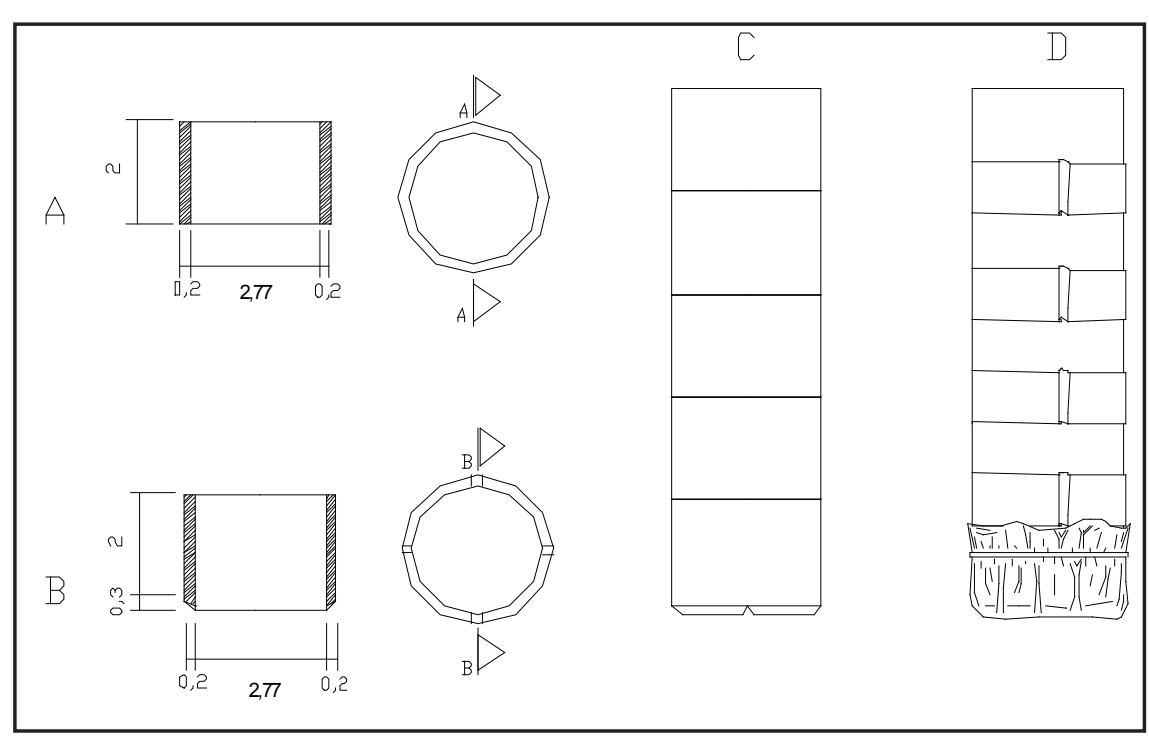

Figura 1. Esquema da coluna apresentando o primeiro anel com $3 \mathrm{~mm}$ de altura a mais (A), fundo em bisel (B), os cilindros sobrepostos (C) e a montagem final da coluna (D) (scheme of column with first ring $3 \mathrm{~mm}$ higher (A), cylinders stacked (B) and cylinders assembly (D)). Campinas, FEAGRI, 2006.

de água dos substratos - Foi observado que o substrato fino de coco reteve mais umidade por unidade de volume, enquanto que o de coco de textura grossa apresentou a menor retenção de água. Independente do tipo de substrato, os de textura fina sempre apresentaram maior retenção de água que os de textura grossa (Figura 2). O substrato fino de coco $(\mathrm{CF})$ apresentou tendência de um mesmo valor de umidade para o ponto de murcha permanente, independentemente da textura. Esse efeito, a priori, pode ser atribuído à porosidade interna das partículas do material que constitui o substrato. Os valores do coeficiente de ajuste das curvas de retenção também em relação ao lençol de água foram encontradas zonas de saturação e franja capilar no substrato. Essas regiões caracterizam-se por terem a microporosidade ocupada por água. Embora essa condição represente o máximo molhamento, estas camadas apresentam um conteúdo de água excessivo, não benéfico às plantas devido à menor aeração. Esse fato pode ser decorrente do maior contato entre partículas do substrato fino, que apresenta uma estrutura matricial que favorece a formação de microporos e maior continuidade no contato entre partículas. Decorrente deste processo, a franja capilar elevou-se acima de 1,5 $\mathrm{cm}$ do lençol de água após uma hora de irrigação. Ao final das 24 horas de saturação, observou-se que a franja capilar saturada permaneceu na camada de $2 \mathrm{~cm}$ iniciais. Por outro lado, a água disponível alcançou aproximadamente nove centímetros de altura, enquanto a água facilmente disponível $5,5 \mathrm{~cm}$. O processo de elevação de água apresentado por esse substrato, é favorável a não-concentração excessiva de sais no perfil do substrato, como Patel (2000) constatou em solos (Figura 3).

O substrato de textura grossa de pinus não apresentou elevação da água disponível dentro do cilindro nos primeiros 15 minutos de saturação, devido provavelmente pelas suas características físicas de textura grossa (Figura 3-B). Para esse tempo de saturação, o conteúdo de água disponível atingiu por volta de dois centímetros de altura em relação ao nível de saturação. Para promover o molhamento de todo o meio de crescimento radicular, torna-se necessário que o nível de saturação esteja localizado até dois centímetros abaixo da superfície do recipiente que o armazena. Não se observou relevante presença de franja capilar, pois essa camada de umidade sob esse nível de tensão se elevou a partir de 30 minutos. Porém durante as 24 horas de avaliação não ultrapassou $0,5 \mathrm{~cm}$ acima do lençol de água. Essa baixa elevação da franja capilar está relacionada à textura do meio, que apresenta menor superfície de contato com a água do recipiente, reduzindo conseqüentemente, a condução da água no meio. Esse fato também pode ser observado na camada de umidade retida sob tensão da franja capilar, que 


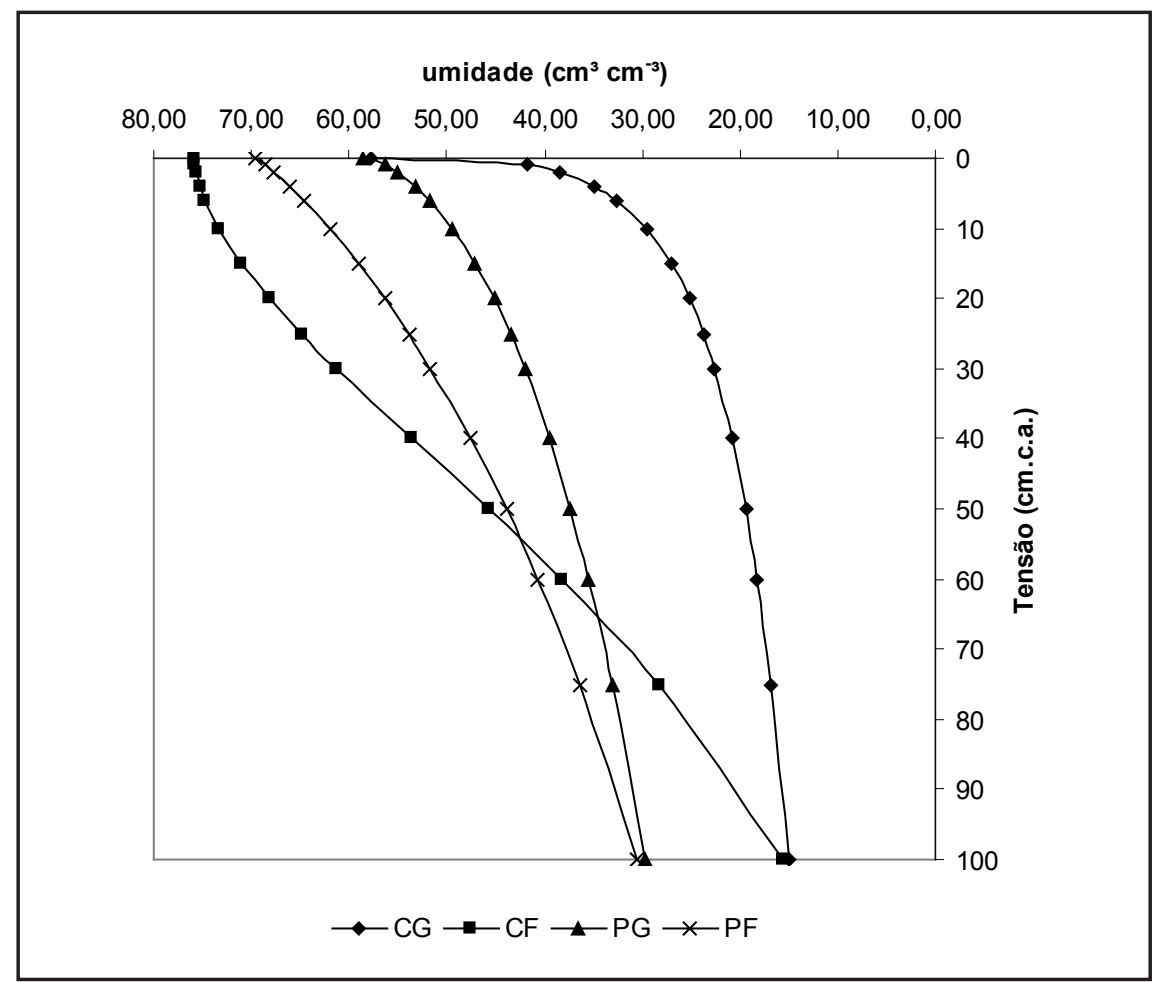

Figura 2. Curva de retenção de umidade do substrato em função do potencial dos substratos fino de coco $(\mathrm{CF})$, coco grosseiro $(\mathrm{CG})$, pinus fino $(\mathrm{PF})$ e pinus grosseiro (PG) (substrate water release for fine fiber coconut $(\mathrm{CF})$, coarse coconut $(\mathrm{CG})$, fine pine bark (PF) and coarse pine bark (PG)). Campinas, FEAGRI, 2007.

não ultrapassou $1 \mathrm{~cm}$ de altura na coluna em 24 horas. A elevação da camada de água facilmente disponível é mais lenta no substrato de textura grossa. O tempo necessário para que a água facilmente disponível atingisse $3,5 \mathrm{~cm}$ de altura foi de 8 horas. Semelhantemente, a camada de umidade sob o nível de energia de água tamponante atingiu $7 \mathrm{~cm}$ de altura após 18 horas de atuação do lençol de água. O substrato fino de pinus permitiu que a água tamponante atingisse $7 \mathrm{~cm}$ entre 1 e 2 horas de atuação do lençol de água.

Comparado aos outros substratos, o pinus de textura grossa obteve menor elevação de água por capilaridade a partir da base da coluna, com menores extratos de água facilmente disponível, discordando dos dados obtidos por Caron et al. (2005b), que afirmaram que a distribuição de tamanho de partículas de substratos não interfere na água facilmente disponível. Devido à granulometria, o substrato de textura grossa exige irrigações mais freqüentes, (De Kreij \& Straver, 1988). Para os substratos de textura grossa, há maior tendência ao ressecamento das camadas favoráveis à formação de uma umidade mais adequada ao desenvolvimento de patógenos. A maior disponibilidade de água pode estar presente na fração com maior microporosidade interna das partículas, o que provavelmente já ocorrera antes dos processos de elevação de água por capilaridade. Fundamentado nas considerações de Patel et al. (2000) para solos, é possível assumir que o substrato fino de coco teria menores possibilidades de acúmulo de sais na superfície.

Foi observada a ausência completa de elevação das linhas de potencial ao longo do tempo (Figura 3-D). No substrato de coco com textura grossa não houve umidade acima da condição de disponível para as plantas, devido ao excesso de fibras longas em um recipiente pequeno, o que promoveu descontinuidade do meio, gerada por aeração excessiva.

O substrato de coco com textura fina apresentou melhor adsorção de água disponível às plantas (Figura 4). Isso ocorre devido à maior eficiência em adsorção de água por massa do substrato de coco. Comparativamente ao substrato fino de pinus, esse atributo seria possibilitado pelas menores densidades do substrato e de suas partículas. A maior capacidade de retenção do substrato de coco, juntamente com menores valores de densidades de partícula e global indica que a microporosidade de dentro das partículas seria a principal característica para o maior conteúdo de água disponível. Por outro lado, a menor capacidade de aumento da adsorção de água ao longo do tempo, demonstra uma condição menos favorável ao transporte de água, devido ao menor contato entre partículas. Essa pode ser a principal causa dos menores volumes de água adsorvidos nesse substrato. Essa retenção de água possibilita maiores conduções de água no meio, fato observado no ganho de água em $69 \%$, entre 15 minutos e 24 horas, valor maior que o observado no substrato de coco, que apresentou $30 \%$. $\mathrm{O}$ substrato de textura grossa de pinus apresentou descontinuidade na adsorção de água em função do tempo, em valores absolutos. Os valores de retenção de água disponível não são concordantes aos observados por Caron et al. (2005b), que verificaram valores entre 0,2 e 0,3 


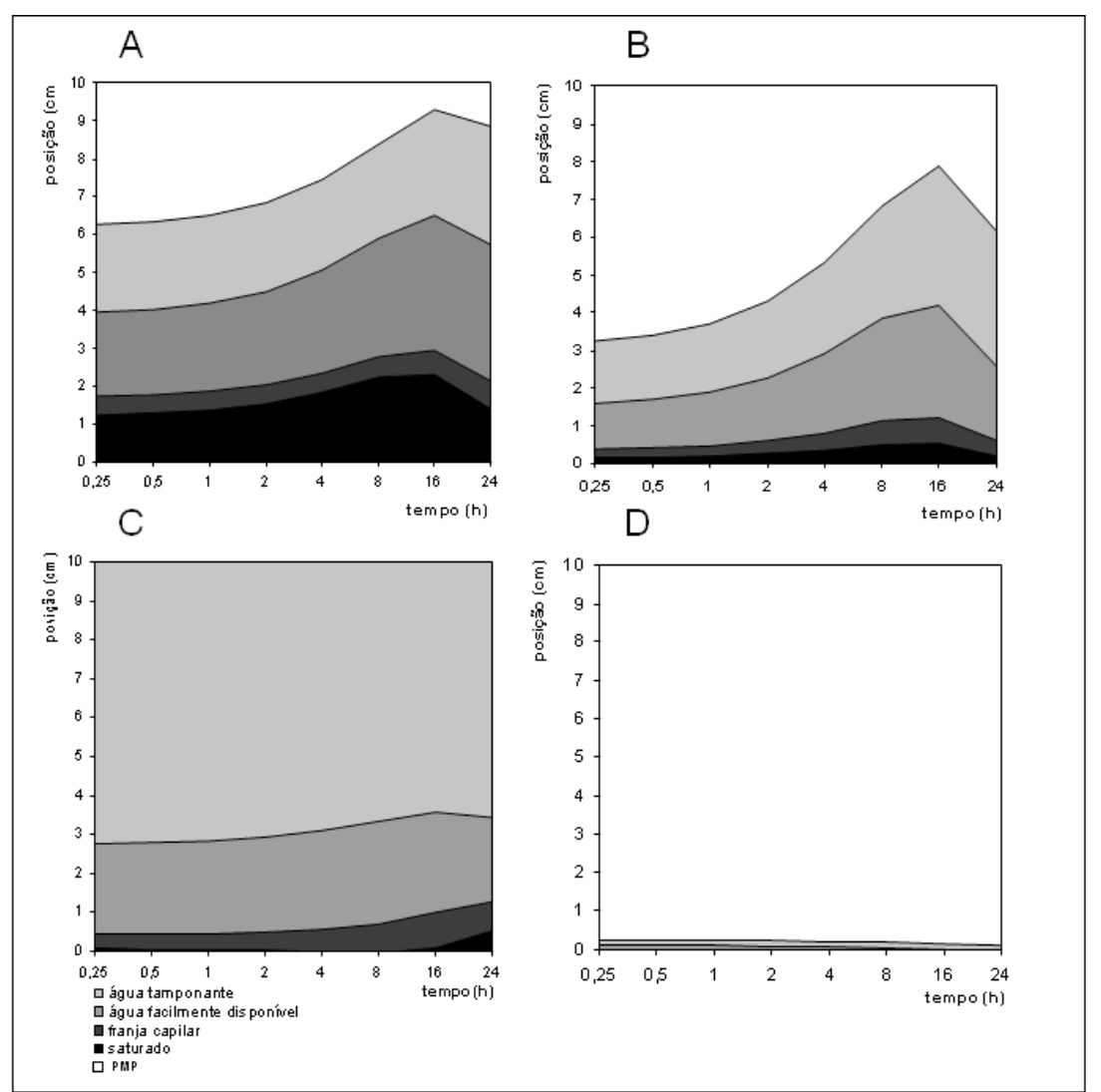

Figura 3. Variação do potencial matricial do substrato fino de pinus ao longo de 24 horas na coluna de $10 \mathrm{~cm}$ de altura, para o substrato fino de pinus (A), grosseiro de pinus (B), fino de coco (C) e grosseiro de coco (D) (variation of matric potential along 24 hours in $10 \mathrm{~cm}$ of column for fine fine pine bark (A), coarse pine bark (B), fine coconut fiber (C) and coarse coconut fiber (D)). Campinas, FEAGRI, 2007.

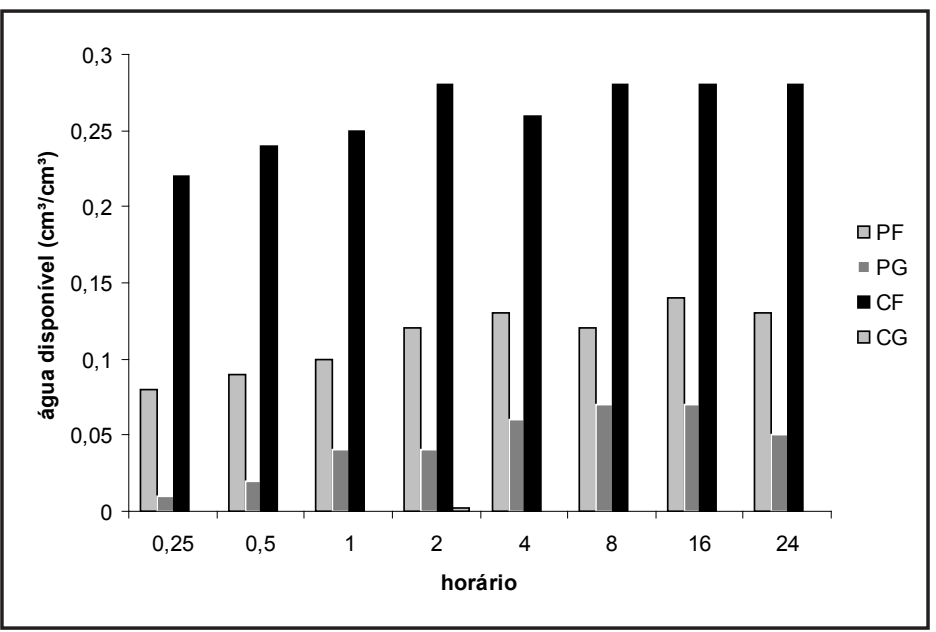

Figura 4. Média de umidade ( $\square$ ) disponível armazenada nas colunas de substrato de pinus textura fina $(\mathrm{PF})$, pinus textura grosseira $(\mathrm{PG})$, coco textura fina $(\mathrm{CF})$ e coco textura grosseira (CG) entre 15 minutos e 24 horas (average available moisture $(\square)$ in substrate columns of fine pine bark (PF), coarse pine bark (PG), fine coconut fiber $(\mathrm{CF})$ and coarse coconut fiber (CG), from 15 minutes to 24 hours). Campinas, FEAGRI, 2007.

$\mathrm{cm}^{3} / \mathrm{cm}^{3}$ de água facilmente disponível armazenada. Essa diferença pode ser decorrente da natureza diferente dos substratos, das práticas de irrigação, da densidade de preenchimento e dos procedimentos para sua realização. Soma-se ao fato de que não há ascensão de água até camadas superficiais do recipiente.

A relação do nível de energia de retenção com a umidade é dependente não apenas da granulometria, mas da natureza das partículas segundo sua porosidade interna, pois esta reflete na habilidade de condução da água no meio pelo processo de adesão, com conseqüências na elevação dos extratos da água facilmente disponível. A textura grossa não favorece a elevação de extratos de água facilmente disponível.

Concluiu-se que ambos os substratos com textura fina apresentam-se adequados para serem utilizados na irrigação por capilaridade, sendo que o substrato de coco, por apresentar maior quantidade de água disponível, mostrou-se o mais recomendado. Adicionalmente, a utilização de substratos com textura grossa se apresenta limitada para elevação de água por capilaridade devido à baixa capacidade de atingir valores adequados de água disponível.

\section{AGRADECIMENTOS}

Ao CNPq pelos recursos disponibilizados para a realização dos trabalhos.

\section{REFERÊNCIAS}

BUNT AC. 1983. Physical properties of mixtures of peats and minerals of different particle size and bulk density for potting substrates. Acta Horticulturae 150: 143-153.

CARON J; ELRICK D. 2005. Measuring the unsaturated hydraulic conductivity of growing media with a tension disc. Soil Science Society American Journal 69: 783-793.

CARON J; ELRICK DE; BEESON R; BOUDREAU J. 2005a. Defining critical capillary rise properties for growing media in nurseries. Soil Science Society American Journal 69: 794-806.

CARON J; RIVIÈRE LM; GUILLEMAIN, G. 2005b. Gas diffusion and air filled porosity: Effect of some oversize fragments in growing media. Canadian Journal of Soil Science 85: 57-65.

DE BOODT M; VERDONCK O; CAPPAERT I. 1974. Method for measuring the water release curve of organic substrates. Acta Horticulturae 37: 2054-2062.

DE KREIJ C; STRAVER N. 1988. Fooded-bench irrigation: effect of irrigation frequency and type of potting on growth of codiaeum and on nutrient accumulation in the soil. Acta Horticulturae 221: 245-252.

GOEBEL MO; BACHMANN J; WOCHE SK; FISHER WR, HORTON, R. 2004. Water 
potential and aggregate size effects on contact angle and surface energy. Soil Science Society American Journal, Madison, 68: 383-393.

LANDIS DL. 1989. Irrigation and water management. In: Landis TD; Tinus, RW; McDonald SE; Barnett JP. The container tree nursery manual, v.4, Agricultural Handbook, 674. Washington, DC U.S. Departament of Agriculture, Forest Service, p.69-188.

NAASZ R; MICHEL JC; CHARPENTIER S. 2005. Measuring hysteretic hydraulic properties of peat and pine bark using a transient method. Soil Science American
Journal 69: 13-22.

PATEL RM; PRASHER SO; BONNEL RB. 2000. Effect of watertable depth, irrigation water salinity, and fertilizer application on root zone salt buildup. Canadian Agricultural Engineering 42: 111-115.

PIRE R; PEREIRAA. 2003. Propriedades físicas de componentes de sustratos de uso común en la horticultura del estado Lara, Venezuela: Propuesta Metodológica. Bioagro 15: 55-63.

RAVIV M; MEDINA S; BEN NER Z; SHAMIR Y. 1993. Very low medium moisture tension - A feasible criterion for irrigation control of container-grown plants. Acta Horticulturae 342: 111-119.

VAN GENUCHTEN MT. 1980. A closedform equation for predicting the hydraulic conductivity of unsaturated soils. Soil. Sci. Soc. Am. J 44: 892-898.

VERDONCK O; PENNINCK R; DE BOODT M. 1983. The physical properties of different horticultural substrates. Acta Horticulturae 150: $155-160$.

YEAGER TH. 1995. Container substrate physical properties. The Woody Ornamentalist, Environmental Horticulture Department, University of Florida, v.20, n.1. 